\title{
Impact of restricting procalcitonin measurements in a Swiss tertiary- care hospital on antibiotic use, clinical outcomes, and costs: An interrupted time-series analysis
}

\author{
Mohamed Abbas MD, MS ${ }^{1,2}$ (1), Nathalie Vernaz PharmD, MBA ${ }^{3}$, Elodie von Dach $\mathrm{PhD}^{4}$, Nicolas Vuilleumier MD, $\mathrm{PhD}^{2,5}$, \\ Stephan J. Harbarth MD, MS ${ }^{1,2,6}$ and Benedikt D. Huttner MD, MS 2,6 \\ ${ }^{1}$ Infection Control Programme, Geneva University Hospitals, Geneva, Switzerland, ${ }^{2}$ Faculty of Medicine, University of Geneva, Geneva, Switzerland, ${ }^{3}$ Finance \\ Department and Medical Direction, Geneva University Hospitals, Geneva, Switzerland, ${ }^{4}$ Clinical Trials Unit, Geneva University Hospitals, Geneva, Switzerland, \\ ${ }^{5}$ Division of Laboratory Medicine, Diagnostic Department, Geneva University Hospitals, Geneva, Switzerland and ${ }^{6}$ Division of Infectious Diseases, Geneva \\ University Hospitals, Geneva, Switzerland
}

\begin{abstract}
We evaluated the impact of a restriction of procalcitonin measurements on antibiotic use, length of stay, mortality, and cost in a Swiss tertiarycare hospital using interrupted time-series analysis. There was no significant change in level or slope for rates of antibiotic consumption, and costs decreased considerably, by $\sim 54,488$ CHF (US $\$ 55,714$ ) per month.
\end{abstract}

(Received 26 May 2020; accepted 24 October 2020; electronically published 2 December 2020)

Appropriate use of antibiotics through antibiotic stewardship is essential to curb the increase in antimicrobial resistance. ${ }^{1,2}$ Clinicians often face uncertainty whether a bacterial infection is present or not in a given patient. Biomarkers such as procalcitonin (PCT), by serving as decisional support, may potentially help avoid unnecessary antimicrobial prescribing. ${ }^{3,4}$

Evidence from meta-analyses of randomized clinical trials (RCTs) suggests that PCT-guided therapy reduces antibiotic use without harm to patients and may even have potential benefits. ${ }^{5}$ The vast majority of these studies were, however, undertaken in patients with lower respiratory tract infections (LRTIs) in the emergency department or sepsis/septic shock in the intensive care unit (ICU). ${ }^{6,7}$

Diagnostic stewardship, that is, the responsible use of diagnostic tests to avoid unnecessary costs and negative consequences associated with false-positive findings, is also a key priority for healthcare systems in an era in which "less is more" and where "smarter medicine" campaigns advocate for rational use of resources. ${ }^{8}$ Overuse and misuse of procalcitonin is frequent in routine practice, ${ }^{9}$ as is the case with many other diagnostic tests. $^{10,11}$ Furthermore, its added value over C-reactive protein remains controversial. ${ }^{12,13}$

Author for correspondence: Dr Mohamed Abbas, E-mail: Mohamed.abbas@hcuge.ch. Or Prof Benedikt Huttner, E-mail: Benedikt. Huttner@hcuge.ch

${ }^{*}$ PREVIOUS PRESENTATION. An abstract of this study was accepted for the $30^{\text {th }}$ European Conference on Clinical Microbiology and Infectious Diseases.

Cite this article: Abbas M, et al. (2021). Impact of restricting procalcitonin measurements in a Swiss tertiary-care hospital on antibiotic use, clinical outcomes, and costs: An interrupted time-series analysis. Infection Control \& Hospital Epidemiology, 42: 890-892, https://doi.org/10.1017/ice.2020.1314
The primary objective of this study was to analyze the effect of restricting PCT measurements on antibiotic use. Secondary objectives were to assess the impact of the restriction on clinical outcomes and costs.

\section{Methods}

This quasi-experimental study was conducted in Geneva University Hospitals (HUG), a tertiary care center in Switzerland. HUG had an antibiotic stewardship program over the entire study period with regularly updated guidelines, review of all positive blood cultures, and dedicated stewardship rounds in certain high-risk wards (eg, ICU). Although the infectious diseases (ID) service issued guidance on the use of PCT (Appendix online), PCT results were not reviewed by the ABS program. The estimated PCT reagent (technical laboratory supplies) cost in our hospital is $\sim 400$ '000 CHF (US $\$ 408,998$ ) per year, which represents $2.4 \%$ of the global annual reagent budget. In contrast, total antibiotic expenditure is $\sim 1.5$ million CHF (US $\$ 1.53$ million) per year. Because of this substantial burden, PCT measurements were restricted as of February 3, 2016, except for the pediatric emergency department, the adult and pediatric ICUs and transplant units. PCT measurements could still be prescribed in other wards with prior approval by an ID consultant and the head of the laboratory division, at their discretion.

We obtained numbers of PCT measurements from the laboratory database as well as patient-level (admission/discharge dates, ward location, in-hospital mortality) and systemic (oral and parenteral) antibiotic administration (ATC class J01 and oral metronidazole) data from the electronic health record system. 
We included patients in all inpatient wards (and the emergency department) in HUG, except those with preintervention low PCT test usage, defined as $<1$ PCT measurement per week, on average, over a 12-month period.

The primary outcome was the change in antibiotic use in defined daily doses (DDD) per 1,000 patient days (PD) per month. The secondary outcomes included days of therapy (DOT) per 1,000 PD per month, in-hospital mortality, length of stay (LOS), and cost savings.

\section{Statistical analysis}

We conducted an interrupted time-series analysis of monthly rates of antibiotic use in DDD per 1,000 PD before and after the start of the intervention using a Prais-Winsten regression, which is based on generalized least-squares method accounting for serial autocorrelation. We used the Durbin-Watson $d$ statistic to evaluate how well the model took into account first-order correlation. We hypothesized the absence of an impact on antibiotic use and overall in-hospital mortality in wards where PCT was discontinued.

We calculated monthly LOS as a ratio of total patient days and number of admissions. In-hospital mortality was calculated as a monthly proportion of deaths by admission. We estimated the cost savings as a percentage reductions of PCT-reagent-related costs and in percentage reductions of the department's global reagent costs. Costs are provided in Swiss Francs (CHF) and US dollars (US\$) with an exchange rate of $1 \mathrm{CHF}=\mathrm{US} \$ 0.978$ (ie, the average exchange rate for 2018, OECD).

The study period was from January 2014 to May 2018, with $>24$ data points on either side of the intervention, which provided sufficient statistical power to analyze the primary outcome. ${ }^{14}$ Data on in-hospital mortality were only available from January 2015 onward. All statistical analyses were performed using Stata version 14 software (StataCorp, College Station, TX) and, in particular, the "itsa" command.

\section{Ethics}

The study was approved by the Ethics Committee of Canton of Geneva (no. 2017-02274), which granted a waiver of informed consent.

\section{Results}

After the intervention, we observed an immediate statistically significant decrease in the level of PCT measurements per month $(-637.4 ; 95 \%$ CI, 539.7-735.0) without a statistically significant change in slope $(-0.05 ; 95 \% \mathrm{CI},-8.2$ to 8.1$)$ (Fig. 1A; Supplementary Tables 1 and 2 online).

Before the intervention, we observed an increasing trend of monthly antibiotic use of 4.3 DDD per 1,000 PD (95\% CI, 1.4-7.1). After the intervention, there was a nonsignificant decrease in level of antibiotic use by 47 DDD per 1,000 PD (95\% CI, -1.0 to 95.6 ), followed by an upward change in slope of $1.6 \mathrm{DDD}$ per $1,000 \mathrm{PD}$ (95\% CI, -0.2 to 3.5 ) which was not statistically significant (Fig. 1B; Supplementary Table 2 online). The Durbin-Watson $d$ statistic changed from 1.25 to 1.67 , indicating that the model performed well despite residual positive autocorrelation. When analyzed by DOT, there was significant decrease in level of 36.6 DOT per 1,000 PD (95\% CI, 2.8-70.3) and a significant decrease in slope of 3.6 DOT per 1,000 PD (95\% CI, 1.0-6.1) after the intervention (Supplementary Table 2 and Supplementary Fig. 1 online).
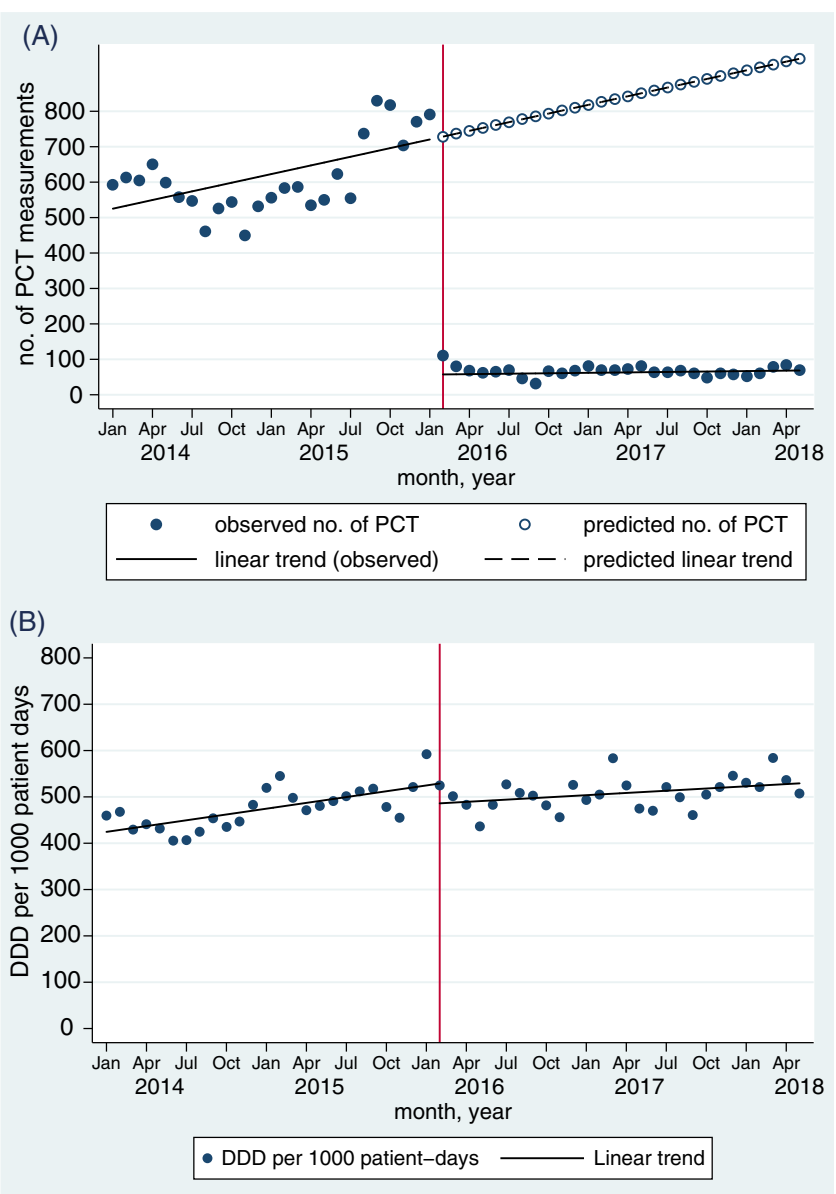

Fig. 1. Changes in (A) monthly number of procalcitonin (PCT) measurements; (B) monthly rate of antibiotic consumption, measured in defined daily doses (DDD) per 1,000 patient days, before and after restriction of PCT measurements.

During the study period, we observed a pre-existing decreasing trend of in-hospital mortality from 7.40 deaths per 100 admissions to 5.02 deaths per 100 admissions $(P<.001)$, with no significant change associated with the intervention (Supplementary Table 2 and Supplementary Fig. 2 online). There was also a pre-existing decreasing trend in LOS from 5.12 to 3.77 days $(P<.001)$ over the study period, with a significant decrease in level after the intervention of 0.4 days (95\% CI, 0.2-0.6) (Supplementary Table 2 and Supplementary Fig. 2 online).

The annual PCT-reagent costs decreased from $\sim 400,000 \mathrm{CHF}$ ( $\sim$ US $\$ 409,000$ ) to 100,000 CHF ( US\$102,000), corresponding to a $75 \%$ decrease. The proportion of PCT reagent costs in the global department reagent budget decreased from $2.4 \%$ to $0.8 \%$.

\section{Discussion}

In this study, the restriction of PCT measurements in a large tertiary-care center in Switzerland did not influence overall antibiotic use and was not associated with increases in LOS or mortality. Meanwhile, the intervention led to significant cost savings.

Although PCT has been repeatedly suggested as means to reduce antibiotic exposure and potentially mortality, mainly in LRTIs, in meta-analyses of RCTs ${ }^{5,15}$ the value of PCT in real-world clinical settings has been questioned. A study including $>20,000$ septic ICU patients suggested that PCT use was associated with increased antibiotic consumption and poorer clinical outcomes, ${ }^{16}$ 
highlighting either the importance of improving implementation of adequate PCT-based strategies prior to widespread adoption or as "worst-case scenario," the limited clinical value of PCT use.

During the study period, we observed pre-existing decreasing trends in mortality and LOS, with no effect of the intervention on in-hospital mortality but a decrease in level of LOS after the intervention. The latter effect may be an artefact; it is unclear how PCT prescription restriction would be associated with decreased LOS. Nevertheless, it is reassuring that we did not observe increases in in-hospital mortality and LOS associated with the intervention.

The main strength of our study was that the "top-down" decision to discontinue PCT measurements offered a natural quasiexperimental study design; this allowed the use of interrupted time-series, which is a robust methodology. ${ }^{17}$ One of the limitations of this study is that it evaluated hospital-level changes in antibiotic prescribing practices and therefore was prone to ecologic bias. Our negative finding does not exclude the possibility that PCT may have an impact on the management of individual patients. Also, we did not evaluate protocol adherence (ie, whether PCT levels influenced prescribing behavior), even though we suspect it to be low, as in other real-world studies. ${ }^{9,18}$ However, since one aim of antimicrobial stewardship is to reduce overall antibiotic use, we feel that the ecologic perspective is still justified. Finally, our single-center study was conducted in a setting where antibiotic use is relatively low; thus, the generalizability of our results may be limited.

Our results do not suggest that PCT is useless; other evidence suggests that when used in specific indications, it may be an effective tool to reduce antibiotic exposure, and thus may be cost-effective. ${ }^{19}$ Appropriate, evidence-based, indications of PCT include (1) initiation of antibiotic therapy in acute LRTI, including communityacquired pneumonia and COPD exacerbation and (2) reducing duration of antibiotic therapy in ICU patients with ventilatorassociated pneumonia or sepsis. Like all diagnostic tests, PCT needs to be used intelligently and in conjunction with other information to have a real impact on patient outcomes. To prevent overuse and misuse of PCT, institutions may need "diagnostic stewardship" teams to enforce restrictions on use or provide real-time review of cases. Further real-life diagnostic-stewardship studies on how to implement PCT-guided therapy are needed to further inform best practices.

Supplementary material. To view supplementary material for this article, please visit https://doi.org/10.1017/ice.2020.1314

Acknowledgments. We would like to thank Dr Angela Huttner (Division of Infectious Diseases, University of Geneva Hospitals, Geneva, Switzerland) for providing input regarding the elaboration of the protocol.

Financial support. This work was partly supported by the Research Fund of the Department of Internal Medicine of the University Hospital and the Faculty of Medicine of Geneva; this Fund received an unrestricted grant from AstraZeneca Switzerland.
Conflicts of interest. All authors report no conflicts of interest relevant to this article.

\section{References}

1. Holmes AH, Moore LS, Sundsfjord A, et al. Understanding the mechanisms and drivers of antimicrobial resistance. Lancet 2016;387:176-187.

2. Dyar OJ, Huttner B, Schouten J, Pulcini C, ESGAP. What is antimicrobial stewardship? Clin Microbiol Infect 2017;23:793-798.

3. Christ-Crain M, Müller B. Procalcitonin in bacterial infections-hype, hope, more or less? Swiss Med Wkly 2005;135:451-460.

4. Neeser O, Branche A, Mueller B, Schuetz P. How to implement procalcitonin testing in my practice. Clin Microbiol Infect 2019;25:1226-1230.

5. Schuetz P, Wirz Y, Sager R, et al. Effect of procalcitonin-guided antibiotic treatment on mortality in acute respiratory infections: a patient level metaanalysis. Lancet Infect Dis 2018;18:95-107.

6. de Jong E, van Oers JA, Beishuizen A, et al. Efficacy and safety of procalcitonin guidance in reducing the duration of antibiotic treatment in critically ill patients: a randomised, controlled, open-label trial. Lancet Infect Dis 2016;16:819-827.

7. van der Does Y, Rood PP, Haagsma JA, Patka P, van Gorp EC, Limper M. Procalcitonin-guided therapy for the initiation of antibiotics in the ED: a systematic review. Am J Emerg Med 2016;34:1286-1293.

8. Lehmann C, Berner R, Bogner JR, et al. The "Choosing Wisely" initiative in infectious diseases. Infection 2017;45:263-268.

9. Garin N, Poffet V, Harbarth S, Perrier A. Antibiotic decision making after procalcitonin measurement in a general internal medicine department: an observational study (abstract A-648-0003-00604). Geneva, Switzerland: European and Swiss Congress of Internal Medicine (ESCIM 2014); 2014.

10. Huang DT, Yealy DM, Filbin MR, et al. Procalcitonin-guided use of antibiotics for lower respiratory tract infection. N Engl J Med 2018;379:236-249.

11. Bremmer DN, Moffa MA, Ma K, et al. Acute exacerbations of chronic obstructive pulmonary disease with a low procalcitonin concentration: impact of antibiotic therapy. Clin Infect Dis 2019;68:725-730.

12. Afshari A, Harbarth S. Procalcitonin as diagnostic biomarker of sepsis. Lancet Infect Dis 2013;13:382-384.

13. Albrich WC, Harbarth S. Pros and cons of using biomarkers versus clinical decisions in start and stop decisions for antibiotics in the critical care setting. Intensive Care Med 2015;41:1739-1751.

14. Bernal JL, Cummins S, Gasparrini A. Interrupted time series regression for the evaluation of public health interventions: a tutorial. Int J Epidemiol 2017;46:348-355.

15. Meier MA, Branche A, Neeser OL, et al. Procalcitonin-guided antibiotic treatment in patients with positive blood cultures: a patient-level metaanalysis of randomized trials. Clin Infect Dis 2019;69:388-396.

16. Chu DC, Mehta AB, Walkey AJ. Practice patterns and outcomes associated with procalcitonin use in critically ill patients with sepsis. Clin Infect Dis 2017;64:1509-1515.

17. de Kraker MEA, Abbas M, Huttner B, Harbarth S. Good epidemiological practice: a narrative review of appropriate scientific methods to evaluate the impact of antimicrobial stewardship interventions. Clin Microbiol Infect 2017;23:819-825.

18. Farooq A, Colón-Franco JM. Procalcitonin and its limitations: why a biomarker's best isn't good enough. J Appl Lab Med 2019;3(4):716.

19. Westwood M, Ramaekers B, Whiting P, et al. Procalcitonin testing to guide antibiotic therapy for the treatment of sepsis in intensive care settings and for suspected bacterial infection in emergency department settings: a systematic review and cost-effectiveness analysis. Health Technol Assess 2015;19(96):v-xxv, 1-236. 